\title{
Conhecimento e Interesse em Ética Médica e Bioética na Graduação Médica
}

\author{
Knowledge and Interest in Medical Ethics and \\ Bioethics in Medical Graduation
}

\author{
Alessandro de Moura Almeida ${ }^{I}$ \\ Almir Galvão Vieira Bitencourt ${ }^{I}$ \\ Nedy Maria Branco Cerqueira Neves ${ }^{I I}$ \\ Flávia Branco Cerqueira Serra Neves ${ }^{I I}$ \\ Marina da Rocha Lordelo ${ }^{I I I}$ \\ Kleuber Moreira Lemos \\ Geila Ribeiro Nuñez ${ }^{I V}$ \\ Marcelo Campos Barbetta \\ Rodrigo Abensur Athanazio ${ }^{I I I}$ \\ Antônio Nery-Filho
}

PALAVRAS-CHAVE:

- Ética Médica;

- Bioética;

- Educação Médica;

- Ensino.

\section{KEY WORDS:}

- Medical Ethics;

- Bioethics;

- Medical Education;

- Learning.

Recebido em: 12/12/2006

Reencaminhado em:27/08/2007

Aprovado em:16/01/2008

REVISTA BRASILEIRA DE EDUCAÇÃO MÉDICA
I Universidade Federal da Bahia, Bahia, Brasil.

"Escola Bahiana de Medicina e Saúde Pública, Bahia, Brasil.

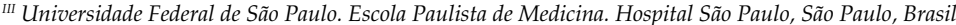

${ }^{I V}$ Hospital Ana Nery. Instituto do coração da Bahia, Bahia, Brasil.

v Universidade Federal da Bahia. Hospital Universitário Professor Edgar Santos, Bahia, Brasil. 


\section{INTRODUÇÃO}

Em virtude das grandes transformações enfrentadas pela medicina nos últimos anos, tornou-se crescente a importância da ética médica e bioética para a compreensão da realidade e antecipação às novas questões impostas na vida daqueles que lidam com a saúde ${ }^{1,2}$. Bioética é uma palavra nova, surgida por volta de 1970, que cristaliza movimentos, aspirações, discursos e práticas que questionam e põem em causa os avanços das técnicas biomédicas. A bioética suscita ainda muitas discussões sobre seu estatuto, métodos e objetivos ${ }^{3}$. Por outro lado, a ética médica é uma forma especial de ética, que se preocupa com os problemas morais da profissão médica ${ }^{4} \mathrm{e}$, assim, pode ser apresentada de forma reduzida à deontologia, ou mais restritamente, à deontologia codificada. Esta dimensão seria mais aplicada à área da saúde, enquanto sua contraposição doutrinária, a bioética, ocuparia um espaço mais amplo, como uma filosofia autônoma, continente de todas as manifestações das cogitações éticas, profissionais ou não, que se passam nas ciências da vida. Entre o espaço ontológico limitado por estes dois extremos, podem se situar numerosas opiniões intermediárias ${ }^{5}$.

Muitos estudiosos têm constatado a discrepância entre o desenvolvimento técnico-científico enfrentado pela profissão médica e a realidade dos consultórios e hospitais no que diz respeito à qualidade do atendimento e à humanização. $\mathrm{Ob}$ serva-se o aumento de denúncias de erro profissional ${ }^{6,7}$, sendo que uma das queixas mais freqüentes dos usuários e dos profissionais de saúde está relacionada à desumanização da prática médica ${ }^{8}$. Além disto, é notada uma separação cada vez maior entre a ética prescrita pelos códigos e aquela exercida pelos profissionais ${ }^{9}$. Tal situação serve como pano de fundo para o aumento dos processos ético-profissionais contra médicos, que alcançam enorme visibilidade em virtude da veiculação pela mídia, concorrendo para o descrédito da figura do médico e a insegurança e revolta dos pacientes ${ }^{10}$. Estes fatos denotam a necessidade de mudanças cada vez mais incisivas no processo de formação dos estudantes de Medicina, na tentativa de reverter este quadro.

A crença de que a formação profissional influencia a conduta do médico e a relação que este estabelece com os pacientes justifica uma análise do panorama do ensino da ética médica e da bioética ${ }^{11-13}$, que tem sofrido grandes transformações nos últimos $30 \operatorname{anos}^{14}$. Somente a partir da década de 1970 as escolas médicas introduziram o ensino da deontologia e diceologia (ética médica) como disciplina autônoma e obrigatória, ainda que ministrada por filosofia moral ou teologia $7,10,15,16$. Antes disso, o ensino se baseava na transmissão passiva e informal de valores e práticas dos professores para os alunos ${ }^{17,18}$. Apesar da franca evolução pela qual vem passando o ensino desta dis- ciplina, muitos ajustes ainda devem ser feitos, entre eles um certo grau de padronização dos modelos pedagógicos.

A necessidade de regulamentação da atividade ocorre porque o médico interage sobre o organismo de terceiros e, desta maneira, poderia haver prevenção de danos. Ética médica poderia ser traduzida, então, pelo zelo com o ser humano ${ }^{19}$. Um dos principais problemas atuais da prática médica diz respeito às dificuldades na relação médico-paciente ${ }^{10,20}$, o que pode comprometer a qualidade do atendimento e a adesão aos tratamentos instituídos. Considerando a ética médica um elemento crucial para o bom estabelecimento da relação médico-paciente, é necessário investigar como está ocorrendo a formação do futuro profissional médico nas faculdades de Medicina. $\mathrm{O}$ objetivo deste trabalho é avaliar o interesse de professores médicos e estudantes do curso médico sobre ética médica e bioética, e seu conhecimento sobre o Código de Ética Médica brasileiro.

\section{MATERIAIS E MÉTODOS}

\section{População e amostra}

Foi realizado um estudo transversal e descritivo, tendo como população de referência estudantes de Medicina regularmente matriculados na Faculdade de Medicina (Famed) da Universidade Federal da Bahia (Ufba, Salvador, Bahia), que já tivessem cursado a disciplina deontologia e diceologia médica (ética médica), oferecida no primeiro semestre da grade curricular obrigatória, e docentes médicos em atividade nessa instituição. Este trabalho foi aprovado pelo Comitê de Ética e Pesquisa da Escola Bahiana de Medicina e Saúde Pública.

A amostra definida foi de $50 \%$ dos alunos de cada semestre, obtida mediante processo sistemático de amostragem. Foram excluídos do estudo os alunos vinculados direta ou indiretamente ao grupo de pesquisa. Devido a perdas e recusas, foi obtida uma amostra de 331 estudantes (34,5\% do total de estudantes regularmente matriculados). Em relação aos professores, o objetivo inicial era realizar um censo, que foi inviabilizado em virtude de perdas e recusas durante o processo de coleta, tendo sido alcançada a participação de 101 docentes médicos $(48,8 \%$ do total).

\section{Coleta de dados}

Como instrumento de pesquisa, foram elaborados dois questionários auto-aplicáveis, um para os professores e outro para os alunos, com questões de múltipla escolha (Anexo). Os questionários estavam divididos em três blocos de perguntas: 1) caracterização do entrevistado (respeitando o anonimato); 2) questões sobre a percepção do ensino de ética médica e bioética; 3) avaliação do conhecimento do Código de Ética Médica 
$(\mathrm{CEM})^{21}$. Os questionários aplicados entre professores e alunos eram similares nos blocos 2 e 3 de questões, diferindo apenas pela presença de uma questão a mais aplicada aos estudantes e que versava sobre seu contato com algum professor que considerassem mau exemplo quanto ao cumprimento do CEM. Os participantes foram solicitados a avaliar a importância da disciplina de ética médica em relação às outras disciplinas e a fazer uma auto-avaliação de seu conhecimento a respeito de ética médica em geral, ambos numa escala de 1 a 5 . O terceiro bloco foi composto por nove questões sobre o conteúdo do CEM.

A coleta de dados foi realizada por integrantes da Academética (Associação de Acadêmicos para o Estudo da Ética Médica e Bioética) ${ }^{22}$, previamente treinados. A abordagem dos professores foi realizada por meio de uma carta de apresentação, durante atividades docentes ou nas reuniões de departamento, na própria Famed - Ufba. A coleta de dados dos estudantes ocorreu durante o processo de matrícula do semestre letivo 2001.2.

\section{Análise de dados}

No processo de análise, foram excluídos os questionários cujas respostas não puderam ser claramente identificadas. Os dados foram expressos como freqüências absoluta e relativa para as variáveis categóricas e como média \pm desvio-padrão (DP) para as variáveis contínuas. Para comparação entre o autoconhecimento em ética médica e bioética, a importância dada à disciplina de ética médica e o total de acertos nas questões do terceiro bloco, foram criados escores, transformandose os valores de cada indivíduo em cada uma dessas variáveis, em escala de 0 a 1 . Dados categóricos foram comparados utilizando-se um teste $\chi 2$ ou o teste exato de Fischer, conforme indicação. Teste $t$ de Student e Anova foram utilizados na comparação de variáveis contínuas. Teste de correlação de Pearson foi utilizado para avaliar a significância das associações entre variáveis ordinais ou contínuas. Valor de $\mathrm{p}$ inferior ou igual a 0,05 foi considerado estatisticamente significante. Os dados foram analisados no programa de análise estatística SPSS, versão 9.0 para Windows (SPSS Inc. Chicago, Illinois).

\section{RESULTADOS}

Foram estudados 101 professores e 331 estudantes. Entre os professores, a idade variou entre 24 e 67 anos, com média de 46,9 $( \pm 8,3)$ anos, sendo $59,4 \%(n=60)$ do gênero masculino. O tempo de formado variou entre 0 e 43 anos, com média de 22,1 $( \pm 8,2)$ anos. $\mathrm{O}$ tempo de ensino variou entre 0 e 39 anos, sendo a média de 17,5 ( $\pm 9,2)$ anos. Entre os alunos, a idade média encontrada foi de 21,6 ( $\pm 2,3$ ), variando entre 18 e 39 anos, sendo $46,8 \%$ (n = 155) do gênero masculino. Quanto ao período que estavam cursando, 39 (11,8\%) estavam no primeiro ano; 79 (23,9\%), no segundo ano; 71 (21,5\%), no terceiro ano; 75 (22,6\%), no quarto ano; 37 (11,2\%), no quinto ano; e 25 (7,5\%), no sexto ano. Cinco alunos não responderam a este campo no questionário.

O momento mais adequado para ministrar a disciplina ética médica seria, na opinião dos estudantes, o primeiro semestre $(28,7 \%)$, seguido da opção "todos os semestres" (21,4\%). Apenas um estudante $(0,3 \%)$ considerou que a disciplina não é essencial durante a graduação e quatro consideraram que a disciplina deveria ser optativa. No grupo dos professores, a maioria $(62,9 \%, n=56)$ considerou que a disciplina deveria ser ministrada em todos os semestres.

Uma parte significante dos professores e dos acadêmicos não se atualiza em bioética ou raramente o faz $(38,1 \%, \mathrm{n}=37$; e $69,6 \%, n=229$, respectivamente). Quanto às fontes de atualização, a mais citada entre os estudantes foi revistas leigas $(34,1 \%)$, seguida por revistas especializadas $(31,1 \%)$, internet $(28,7 \%)$, e eventos científicos $(21,5 \%)$. Pôde-se observar que houve um equilíbrio entre as diversas fontes de atualização deste grupo. Contudo, houve um predomínio de revistas especializadas $(66,0 \%)$ como fontes de atualização entre os professores estudados, seguidas por eventos científicos (41,2\%) e internet (38,1\%). Apenas 9,3\% dos professores afirmaram utilizar revistas leigas como fontes de atualização.

A maioria dos professores $(86,2 \%, n=87)$ refere ter lido o Código de Ética Médica pelo menos parcialmente nos últimos dez anos. No entanto, 12 (11,9\%) responderam não ter lido o CEM neste período, e 2 não especificaram sua leitura do CEM. Todos os acadêmicos disseram ter lido o CEM pelo menos parcialmente, sendo que $48,6 \%$ o leram em sua totalidade.

Ao serem perguntados sobre a importância atribuída à disciplina ética médica, quando comparada às outras disciplinas, a média de importância, numa escala de 1 a 5, atribuída por professores e estudantes foi de $4,7( \pm 0,7)$ e de $4,5( \pm 0,8)$, respectivamente $(\mathrm{p}=0,086)$. A auto-avaliação sobre o conhecimento sobre ética médica em geral, também numa escala de 1 a 5 , foi de $3,4 \pm 0,9$ para os professores e de 3,2 $\pm 0,7$ para os estudantes $(p=0,017)$. Além disso, foi observado que a autoavaliação do conhecimento sobre ética médica foi inferior à importância dada ao tema tanto no grupo dos professores $(\mathrm{p}<$ $0,001)$, quanto no grupo dos estudantes $(p<0,001)$.

A análise dos dados referentes à avaliação do conhecimento do CEM, testado no terceiro bloco do questionário, é demonstrada na Tabela 1, tanto para os professores quanto para os estudantes. Do total de nove questões, a média de acertos foi de 5,0 $\pm 1,9$ questões para os professores e de 5,9 $\pm 1,5$ para os acadêmicos ( $<<0,001)$. Não foi observada diferença estatística entre os diferentes semestres quanto à média de acertos no grupo dos estudantes. Entre os professores, o tempo de for- 
TABELA 1

Respostas corretas no terceiro bloco do questionário entre professores médicos e estudantes da Famed - UFBA

\begin{tabular}{|c|c|c|}
\hline \multirow{2}{*}{ QUESTÃO } & \multicolumn{2}{|c|}{ Resposta correta } \\
\hline & Professores & Estudantes \\
\hline O CEM normatiza relações entre médicos, médicos e enfermeiros, médicos e outros profissionais de saúde. & $78(77,2 \%)$ & $310(93,7 \%)$ \\
\hline Médicos, enfermeiros e outros profissionais da área de saúde estão submetidos ao CEM. & $55(54,5 \%)$ & $225(68,0 \%)$ \\
\hline O CEM é punitivo. & $20(19,8 \%)$ & $108(32,6 \%)$ \\
\hline O CEM trata diretamente sobre a barriga de aluguel. & $48(47,5 \%)$ & $157(47,4 \%)$ \\
\hline O CEM trata diretamente sobre o sigilo profissional. & $89(88,1 \%)$ & $320(96,7 \%)$ \\
\hline O CEM trata diretamente sobre a greve dos médicos. & $32(31,7 \%)$ & $212(64,0 \%)$ \\
\hline O CEM trata diretamente sobre a Aids. & $50(49,5 \%)$ & $140(42,3 \%)$ \\
\hline O CEM proíbe a clonagem de seres humanos. & $50(49,5 \%)$ & $179(54,1 \%)$ \\
\hline Ao prescrever uma receita com a grafia ilegível, o médico infringe um artigo do CEM. & $82(81,2 \%)$ & $297(89,7 \%)$ \\
\hline
\end{tabular}

mado e o tempo de ensino também não influenciaram o seu conhecimento sobre questões do CEM. A questão com maior número de acertos em ambos os grupos foi a que tratava sobre o sigilo profissional, enquanto a com menor número de acertos, também em ambos os grupos, foi a que versava sobre o caráter punitivo do CEM (Tabela 1).

A média de acertos no terceiro bloco do questionário foi relacionada positivamente com a leitura do CEM nos últimos dez anos, tanto para alunos ( $\mathrm{p}=0,002)$, quanto para professores $(p=0,006)$. Além disso, verificou-se que, para os professores, quanto maior a freqüência de atualização, maior a média de acertos na avaliação sobre o conhecimento do CEM (nunca $=2,50 \pm 1,73$; raramente $=4,48 \pm 1,54 ;$ anualmente $=4,47 \pm$ 1,58 ; semestralmente $=5,32 \pm 1,63$; mensalmente $=6,14 \pm 2,44$; $\mathrm{p}=0,001)$. Embora tal comportamento também se repetisse entre os estudantes, a diferença encontrada não foi estatisticamente significante (nunca $=5,56 \pm 1,37$; raramente $=5,83 \pm$ 1,45 ; anualmente $=6,13 \pm 1,16$; semestralmente $=6,04 \pm 1,94$; mensalmente $=6,22 \pm 1,52 ; \mathrm{p}=0,412$ ).

A Tabela 2 mostra uma comparação entre os dados de importância atribuída à disciplina ética médica, auto-avaliação sobre o conhecimento de ética médica e conhecimento do CEM. Não foi observada correlação entre a importância dada à disciplina e o número de acertos em nenhum dos grupos estudados. Contudo, o número de acertos mostrou correlação com o escore de autoconhecimento em ambos os grupos $(p<0,01)$.

Quando questionados sobre o comportamento de seus professores em relação ao cumprimento do CEM, 70,6\% dos estudantes afirmaram ter observado conduta inadequada de seus preceptores no atendimento de pacientes. Além disso, como demonstrado no Gráfico 1, este resultado é influenciado pelo momento do curso em que o estudante entrevistado se encontra. Contudo, o contato com este professor considerado
TABELA 2

Comparação entre importância atribuída à disciplina ética médica, auto-avaliação sobre o conhecimento de ética médica em geral e número de acertos no bloco de questões sobre conhecimento do Código de Ética Médica por estudantes e professores

\begin{tabular}{lccc}
\hline Grupo & Acertos * & Auto-avaliação * & Importância * \\
\hline Estudantes & $0,6239 \pm 0,1666$ & $0,6354 \pm 0,1398$ & $0,9058 \pm 0,1546$ \\
Professores & $0,5545 \pm 0,2163$ & $0,6760 \pm 0,1724$ & $0,9354 \pm 0,1335$ \\
\hline
\end{tabular}

* Dados expressos em média \pm desvio padrão.

Professores: acertos x importância: $\quad \mathrm{p}<0,001$ acertos $x$ auto-avaliação: $p<0,001$

Estudantes: acertos $\mathrm{x}$ importância: $\mathrm{p}<0,001$ acertos $x$ auto-avaliação: $p=0,122$

"mau exemplo" não alterou o conhecimento do estudante sobre o CEM, a auto-avaliação de seu conhecimento sobre ética médica e bioética, e a importância dada por ele à disciplina de ética médica.

\section{GRÁFICO 1}

Contato dos estudantes com professores que consideram mau exemplo em relação ao cumprimento do CEM.

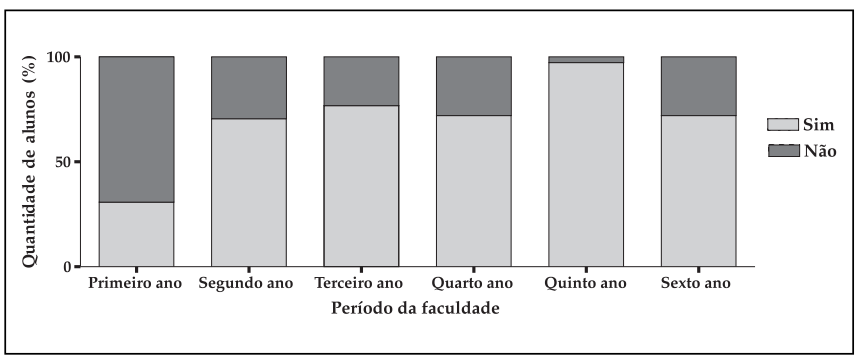




\section{DISCUSSÃO}

Nas últimas décadas, o ensino da ética médica e bioética se tornou fundamental nas faculdades de Medicina de todo o mundo, com o objetivo de formar profissionais dignos e condizentes com as expectativas da sociedade ${ }^{14,19}$. A importância atribuída a esta disciplina tem crescido, e a tendência é que o ensino de ética médica seja ampliado para todo o curso médico, enfatizando as discussões de tópicos éticos e legais controversos $^{23}$. Desta maneira, é possível tornar os estudantes capazes de enfrentar os complexos dilemas do dia-a-dia, com os quais terão que se confrontar durante a prática médica ${ }^{15,24}$. Entretanto, para alcançar este objetivo, é necessário que professores e alunos se empenhem neste tipo de aprendizado. No presente trabalho, foi avaliado o interesse de discentes de Medicina e docentes médicos no ensino destes saberes e podemse destacar alguns pontos.

A maioria dos professores e grande parte dos alunos concordaram em que a disciplina deveria ser ministrada em todos os semestres da graduação, conforme destacado em 1983 pelo estudo de Olukoya ${ }^{25}$. O ensino pontual em apenas um semestre parece ter pouco efeito na formação dos estudantes. Shorr $e t$ al. ${ }^{26}$, avaliando conhecimentos e atitudes sobre aspectos éticos de acadêmicos de Medicina do primeiro ano, não encontraram diferença no padrão de resposta quando os mesmos foram avaliados antes e após o curso oferecido pela faculdade. $\mathrm{O}$ autor justifica tal achado pelas perspectivas éticas bem estabelecidas dos estudantes no momento de entrada no curso. Contudo, já foi demonstrado um declínio na atitude dos alunos ao longo do curso, que pode estar relacionado à perda do idealismo ou a outros fatores inerentes ao impacto do currículo em suas vi$\operatorname{das}^{27}$, bem como à mudança de seus valores pessoais ${ }^{28}$.

A realidade do ensino atual no Brasil, segundo Munõz e Munõz ${ }^{7}$, é que, na maior parte (76,1\%) das escolas médicas brasileiras, a disciplina é abordada em apenas um semestre, sendo principalmente no quarto ano $(27,8 \%)$. Esse mesmo estudo verificou que apenas três faculdades (3,1\%) ensinam a disciplina durante os seis anos do curso ${ }^{7,19}$. Embora tenha sido demonstrado que a maioria dos alunos considera adequado o ensino da disciplina durante o primeiro ano do curso médico, há evidências da consciência da necessidade de sua extensão a todos os semestres. Na faculdade onde este estudo foi realizado, à época da coleta dos dados, a matéria era ministrada no primeiro semestre, na disciplina de deontologia e diceologia médica. Recentemente, durante a reforma curricular, foi criado um eixo temático ético e humanístico, de forma a estender o ensino dessa disciplina a todo o curso. Um trabalho que envolveu 91 escolas médicas dos Estados Unidos e Canadá, realizado por Lehmann et al. ${ }^{29}$ em 2000, revelou que 55\% das faculdades ti- nham um curso introdutório de ética médica; em 78\% delas, a disciplina era parte de outras matérias no período pré-clínico; em $74 \%$ delas, a disciplina era oferecida durante o período clínico. Este resultado representou um aumento de quase duas vezes no ensino durante o período clínico do curso, em relação a outro trabalho realizado nesses países em $1985^{30}$

Tanto estudantes como professores atribuem elevada importância à disciplina, comparada às outras disciplinas, para sua formação como médico. Diversos trabalhos demonstram que os estudantes percebem a necessidade de uma formação que contribua para a gênese de uma competência moral ${ }^{31,32}$. Olukoya et al. ${ }^{25}$ demonstraram, há mais de 20 anos, que $88 \%$ dos estudantes acreditavam que a ética médica tinha lugar em seus currículos, e $84 \%$ classificaram como alta sua importância numa boa prática clínica.

No conteúdo da disciplina trabalha-se mais com argumentos e menos com verdades, daí a necessidade de promover discussões. Dessa forma, é difícil afirmar qual o melhor método de avaliação, devido à complexidade de medir posturas e comportamentos que, muitas vezes, envolvem valores e crenças ${ }^{19}$. Avaliar o conhecimento sobre o teor da disciplina é uma tarefa difícil, que não pode ser feita apenas com base no conteúdo do CEM. No entanto, visto que este documento rege a conduta profissional dos médicos em nosso meio, é fundamental avaliar seu conhecimento tanto pelos docentes médicos, responsáveis por transmitir estes saberes aos futuros médicos, quanto por estes, sujeitos mais tarde à fiscalização. Neste trabalho, os estudantes tiveram um desempenho melhor, com maior média de acertos do que os professores, embora ambos os grupos tivessem médias de acertos consideradas regulares, de $62,4 \%$ e $55,5 \%$, respectivamente. Tal resultado pode ser justificado, em parte, pela evidência de que uma parcela significativa dos professores médicos não se atualiza e uma parte nem sequer leu o CEM nos últimos dez anos. De igual forma, é baixo o conhecimento relatado nos estudos de Shiraz $^{33}$ e Sulmasy ${ }^{34}$. Shiraz ${ }^{33}$, avaliando a leitura do Código de Ética Médica entre médicos cirurgiões, encontrou que apenas $44 \%$ da amostra estudada leram o código parcial ou completamente. Esses resultados apontam um desinteresse dos docentes, em geral, pelos temas estudados.

Eckles e colaboradores ${ }^{35}$ recomendam dois pontos quanto à finalidade do ensino da disciplina: formar médicos virtuosos e promover um conjunto de habilidades e competências para analisar e resolver dilemas éticos. Estas considerações refletem os resultados dos currículos da disciplina, muito mais difíceis, teórica e empiricamente, de serem caracterizados e medidos do que nas outras áreas. Nessa vertente, o estudo sugere que os dados encontrados refletem esta argumentação através da 
dificuldade de resolução de conflitos, ao tempo em que se observa também a dificuldade em mensurar os conteúdos que se devem utilizar para atingir os objetivos propostos. Vale ressaltar que uma das questões propostas por Eckles e colegas se refere à percepção dos estudantes de Medicina quanto aos problemas éticos que foram contemplados e analisados no presente estudo.

Entre as questões do terceiro bloco do questionário, a que obteve menor índice de acerto, tanto para alunos como professores, foi a que versava sobre o caráter punitivo do CEM. O código não trata diretamente de penalidades, mas busca oferecer os postulados fundamentais ao exercício ético da profissão médica, de forma que infrações a estas normas são passíveis de julgamento e punição, de acordo com o Código de Processo Ético-Profissional (CFM no 1.617/01) ${ }^{36}$. Estudantes de Medicina, obviamente, não estão sujeitos ao CEM, mas conduzem seu aprendizado junto aos pacientes e enfrentam dilemas éticos em sua prática diária. Com base neste preceito, foi recentemente apresentada uma Proposta de Código de Ética dos Estudantes de Medicina da Ufba, com normas adaptadas do CEM e que visam orientar o estudante frente a situações cotidianas do curso, de forma a prepará-lo para o exercício da profissão ${ }^{37}$.

Outro resultado preocupante e que vai de encontro à importância atribuída à disciplina é a baixa freqüência de atualização neste tema por alunos, principalmente, mas também por grande parte dos professores entrevistados. Nos últimos anos, grande destaque tem sido dado à educação médica continuada, permitindo ao profissional médico se atualizar sobre novas técnicas ${ }^{24}$. Para este fim, a inserção de temas éticos nos congressos de especialidades, fóruns e simpósios regionais tem sido muito freqüente no País, tal como a veiculação de diversos artigos nas principais revistas nacionais e internacionais ${ }^{38}$. Esta necessidade de atualização parte também da própria sociedade, visto que estes temas têm sido colocados em pauta com freqüência principalmente pela mídia não especializada.

Para os estudantes, é ainda mais importante estar atentos às discussões éticas atuais e não se limitar ao programa da disciplina. Neste contexto, vale destacar a iniciativa de um grupo de estudantes de Medicina da Bahia que, com o apoio dos professores de duas faculdades locais e do Conselho Regional de Medicina do estado da Bahia (Cremeb), fundou a Associação de Acadêmicos para o Estudo da Ética Médica e Bioética (Academética), no intuito de dar continuidade ao aprendizado nesta área por meio de palestras, conferências, filmes, discussão de artigos e pesquisa ${ }^{22}$.

O docente deve possibilitar o aprendizado de habilidades psicomotoras, ao tempo em que fornece parâmetros socialmente justos e eticamente aceitos de relacionamento médico- paciente $^{39}$. A formação ética dos futuros profissionais da medicina pode ser influenciada pelo exemplo dos professores; condutas inadequadas contribuem para a formação de médicos sem compromisso com os princípios éticos ${ }^{20,40,41}$. Satterwhite et al. ${ }^{20}$ reportaram que $35 \%$ dos estudantes do primeiro ano de uma faculdade dos Estados Unidos eram expostos a comportamento antiético de médicos e residentes, e este percentual aumentou para $90 \%$ nos alunos do quarto ano. Em outro artigo publicado em 2000, os mesmos autores relataram que 35\% daqueles estudantes afirmaram que comentários depreciativos feitos por residentes na ausência ou presença dos pacientes eram, algumas vezes, considerados apropriados, sendo que a proporção de estudantes com esta opinião aumentava durante o curso de $24 \%$ no primeiro ano para $55 \%$ no quarto ano ${ }^{20}$. Fuedtner et al. ${ }^{42}$ revelaram que os acadêmicos são freqüentemente expostos por seus preceptores a situações em que se sentem obrigados a participar de atos que julgam antiéticos. Este estudo revelou que $58 \%$ dos entrevistados relataram ter realizado alguma ação considerada antiética e $62 \%$ acreditam que pelo menos alguns de seus princípios éticos já haviam sido maculados ou perdidos durante o curso.

Em nossa casuística, percebemos que grande parte dos estudantes julga ter encontrado, durante o curso de graduação, professores considerados maus exemplos e que este contato aumentou ao longo do curso. Esse dado merece especial atenção dos educadores e da comunidade acadêmica, uma vez que o próprio curso de Medicina pode estar contribuindo para uma deterioração na formação moral de seus estudantes. Ensinar é uma atividade política e ética que exige grande responsabilidade docente, pois os alunos se miram em seus mestres e anseiam por seus saberes ${ }^{43}$.

\section{CONCLUSÃO}

O estudo realizado revela que professores médicos e estudantes de Medicina consideram ética médica e bioética extremamente importantes, apesar das evidências de baixo interesse, atualização e conhecimento.

Chama a atenção, ainda, a referência feita pelos alunos a professores considerados maus exemplos no cumprimento do CEM e sua repercussão na formação dos futuros profissionais.

Por se tratar de um tema pouco estudado, este trabalho fornece dados originais em nosso meio sobre a percepção que alunos e professores têm da disciplina, contribuindo para melhor fundamentação do ensino médico em sua vertente humanista, fundamental à prática profissional.

Finalizando, sugere-se a realização de novos estudos acerca de quem deve ensinar, quais os melhores métodos de ensino e maneiras de avaliar. 


\section{AGRADECIMENTOS}

Gostaríamos de agradecer a todos os que contribuíram direta ou indiretamente para a execução deste trabalho, ao Conselho Regional de Medicina do Estado da Bahia (Cremeb), pelo apoio, e a todos os membros da Academética, em especial Alana Soares, Ana Claúdia Ramos, Dayanne Fonseca, Maria Ignez Braghiroli, Monalisa Cunha e Ivana Maria Santos (in memoriam).

\section{REFERÊNCIAS}

1. Diniz D, Guilhem DB, Garrafa V. Bioethics in Brazil. Bioethics 1999;13(3-4):244-8.

2. Potter VR. Bioethics: bridge to the future. [S.1.]: Englewood Cliffs; Prandice-Hall, 1971.

3. Canto-Sperber M. Dicionário de ética e filosofia moral. São Leopoldo, RS: Ed. UNISINOS, 2003.

4. Lopes JL. A Ética Médica na formação profissional de Medicina. Rev Bras Educ Med 1980; 4(2): 45-52.

5. Sá Junior LSM. Ética do professor de Medicina. Bioética 2002; 10(1): 49-84.

6. Miyasaka M, Akabayashi A, Kai I, Ohi G. An international survey of ethics curricula in Asia. J Med Ethics 1999; 25: 514-21.

7. Muñoz D, Muñoz DR. O ensino da ética nas faculdades de medicina do Brasil. Rev Bras Educ Med 2003; 27(2): 114-24.

8. Rego S, Schramm FR, Garbayo LS, Almeida JLT. Proposta de um selo de humanização para os hospitais brasileiros. Relatório de pesquisa. Rio de Janeiro: Ensp; 2001.

9. Berlinguer G. Ética da saúde. São Paulo: Hucitec; 1996.

10. Grisard N. Ética Médica e Bioética: a disciplina em falta na graduação médica. Bioética 2002; 10(1): 97-114.

11. Price J, Price D, Williams G, Hoffenberg R. Changes in medical student attitudes as they progress through a medical course. J Med Ethics 1998;24(2):110-7.

12. Myser C, Kerridge IH, Mitchell KR. Teaching clinical ethics as a professional skill: bridging the gap between knowledge about ethics and its use in clinical practice. J Med Ethics 1995;21(2):97-103.

13. Yacoub AA, Ajeel NA. Teaching medical ethics in Basra: perspective of students and graduates. East Mediterr Health J 2000;6(4):687-92.

14. Fox E, Arnold RM, Brody B. Medical ethics education: past, present, and future. Acad Med 1995;70(9):761-9.

15. Glick S. The teaching of medical ethics to medical students. J Med Ethics 1994; 20: 239-43.

16. D'Avila R. É possível ensinar Ética Médica em um curso formal curricular? Bioética 2002; 10(1): 115-26.

17. Parker M, Price DA, Harris PG. Teaching of medical ethics: implications for an integrated curriculum. Med Educ 1997; 31: 181-7.
18. Lovett L, Seedhouse D. An innovation in teaching ethics to medical students. Med Educ 1990; 24: 37-41.

19. Neves N. Avaliação do Ensino de Ética Médica nas Escolas Médicas de Salvador-Bahia: Elementos contributivos para a Humanização da Medicina [Dissertação]. Universidade Federal da Bahia, 2005.

20. Satterwhite WM, Satterwhite RC, Enarson CE. Medical students' perceptions of unethical conduct at one medical school. Acad Med 1998;73(5):529-31.

21. Conselho Federal de Medicina. Código de Ética Médica, Resolução CFM n 1246/88. Brasília (DF): CFM, 1988.

22. Athanazio RA, Lemos KM, Fonseca DC, Cunha MS, Braghiroli MIFM, Almeida AM, et al. Academética: Um Novo Método de Estudo Continuado sobre Ética Médica e Bioética. Rev Bras Educ Med 2004; 28(1): 73-8.

23. Goldie J, Schwartz L, McConnachie A, Morrison J. The impact of a modern medical curriculum on students' proposed behavior on meeting ethical dilemmas. Med Educ 2004; 38(9): 942-9.

24. Wlasienko P. Ethical and legal aspects in teaching students of medicine. Sci Eng Ethics 2005; 11(1): 75-82.

25. Olukoya AA. Attitudes of medical students to medical ethics in their curriculum. Med Educ 1983; 17(2): 83-6.

26. Shorr AF, Hayes RP, Finnerty JF. The effect of a class in medical ethics on first-year medical students. Acad Med. 1994;69(12):998-1000.

27. Woloschuk W, Harasym PH, Temple W. Attitude change during medical school: a cohort study. Med Educ 2004; 38(5); 522-34.

28. Satterwhite RC, Satterwhite WM, Enarson CE. An ethical paradox: the effect of unethical conduct on medical students' values. J Med Ethics 2000;26:462-465.

29. Lehmann LS, Kasoff WS, Federman DD. A Survey of Medical Ethics Education at U.S. and Canadian Medical Schools. Acad Med 2004; 79: 682-689.

30. Bickel J. Integrating Human Values Teaching Programs into Medical Students' Clinical Ed ucation. Project Report to the AAMC. Washington, DC: Association of American Medical Colleges, 1986.

31. Taquette SR, Rego S, Schramm FR, Soares LL, Carvalho SV. Situações eticamente conflituosas vivenciadas por estudantes de medicina. Rev Assoc Med Bras 2005; 51(1): 23-8.

32. Goldie J, Schwartz L, McConnachie A, Morrison F. The impact of three years' ethics teaching, in an integrated medical curriculum, on students' proposed behaviour on meeting ethical dilemmas. Med Educ 2002; 36: 489-97.

33. Shiraz B, Shamim MS, Shamim MS, Ahmed A. Medical ethics in surgical wards: knowledge, attitude and practice 
if surgical team members in Karachi. Indian J Med Ethics 2005; 2(3): 94-6.

34. Sulmasy DP, Dwyer M, Marx E. Knowledge, confidence, and attitudes regarding medical ethics: how do faculty and housestaff compare? Acad Med 1995; 70(11): 1038-40.

35. Eckles R, Meslin EM, Gaffney M, Helft PR. Medical ethics education: Where are we? Where should we be going? Acad Med 2005; 80(12): 1143-52.

36. Conselho Federal de Medicina. Código de Processo Ético Profissional, Resolução CFM n 1716/01. Brasília (DF): CFM, 2001.

37. Lemos K, Neves N, Athanazio R, Lordelo M, Bitencourt A, Neves FS, et al. Proposta de Código de Ética dos Estudantes de Medicina da Bahia. Gaz Med Bahia 2005; 75(2): 133-42.

38. Gomes JCM. O Atual Ensino da Ética para os Profissionais de Saúde e seus Reflexos no Cotidiana do Povo Brasileiro. Bioética [serial on line] 1996 [cited 2006 Mar 20]; 4(1): [1 screen]. Available from: URL: http://www.portalmedico. org.br/ revista/bio1v4/atualens.html.

39. Siqueira JE, Sakai MH, Eisele RL. O ensino da ética no curso de Medicina: a experiência da Universidade Estadual de Londrina (UEL). Bioética 2002; 10(1):85-95.

40. Testerman JK, Morton KR, Loo LK, Worthley JS, Lamberton $\mathrm{HH}$. The natural history of cynicism in physicians. Acad Med 1996;71:S43-45.
41. Neitzke G. Teaching medical ethics to medical students: moral, legal, psychological and philosophical aspects. Med Law 1999; 18: 99-105.

42. Fuedtner C, Christakis DA, Christakis NA. Do clinical clerks suffer ethical erosion? Students' perceptions of their ethical environment and personal development. Acad Med 1994;69:670-679.

43. Nicholas B. Power and the teaching of medical ethics. J Med Ethics 1999; 25(6): 507-13.

ANEXO: O questionário utilizado está disponível para consulta em http:/ / www.educacaomedica.org.br/Anexos

\section{CONFLITOS DE INTERESSE}

Declarou não haver

\section{ENDEREÇO PARA CORRESPONDÊNCIA}

Alessandro de Moura Almeida

Rua Nossa Senhora de Brotas, 107 - Fauna, 703

Brotas - Salvador

CEP 40285-70 / BA

E-mail: alessandrodemoura@yahoo.com.br 